\title{
Ergodic Pumping: \\ a mechanism to drive biomolecular conformation changes
}

\author{
RS MacKay \\ Mathematics Institute, University of Warwick, \\ Coventry CV4 7AL, UK \\ DJC MacKay \\ Cavendish Laboratory, University of Cambridge, \\ Cambridge CB3 0HE, UK
}

Submitted to Physica D, 30th June 2005. Corrected 10th September 2005.

\begin{abstract}
It is proposed that a significant contribution to the power stroke of myosin and similar conformation changes in other biomolecules is the osmotic pressure of a single molecule (e.g. a phosphate ion) expanding a trap.
\end{abstract}

\section{Introduction}

Conformation changes are an important feature of much biochemistry, e.g. G-proteins, ion channels, photoreceptors, chaperonins, haemoglobin, and of particular interest to us, biomotors, especially myosin. For surveys of biomotors see [How, HuS, HTS]. What are the design principles that evolution has discovered for making efficient molecular motors?

Many mechanisms have been proposed for driving conformation changes. Some have received strong experimental support and wide acclaim (e.g. ATP-synthase [Bo]). Most proposals focus on binding of ligands as the driving force. The basic requirement, however, is just a decrease in free energy [Hill]. Thus, in principle, release of ligands could also drive conformation changes if the departing ligands gain enough entropy to compensate for their loss of binding energy. Indeed, release of ligands must occur at some stage in any cycle of conformation change involving binding of ligands, so its effects must be considered.

Release of ligands can decrease free energy by increasing entropy. For example, opening a trap of small volume $v_{0}$ containing one molecule of an ideal gas into a large container with density $\rho=1 / v_{1}$ molecules per unit volume (figure 1a) increases the entropy by approximately $k \ln \frac{v_{1}}{v_{0}}$ ( $k$ being Boltzmann's constant). This can be quite large [HMWZ, PJ]. Taking rough values for the volume $v_{0}=1 \AA^{3}$ available to the centre of mass of a phosphate ion in the ATP binding pocket of myosin after hydrolysis, and concentration $\rho=2 \mathrm{mM}$ in the cellular solution outside (while fully conscious that phosphate ions in solution do not form an ideal gas), we find $\Delta S=13 k$ for the release of the phosphate ion, and so $\Delta G=13 k T$ at temperature $T$. If such a 


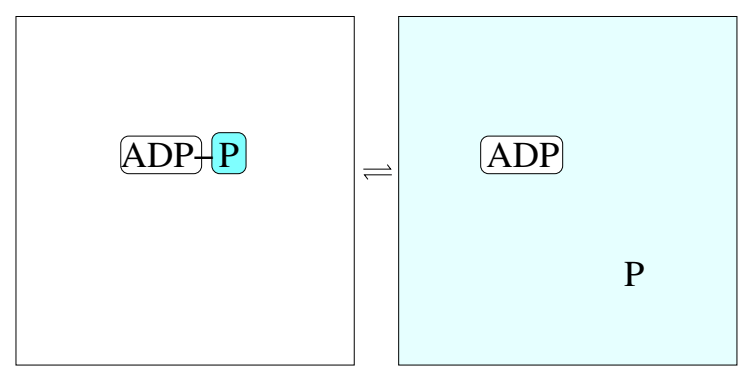

(a)

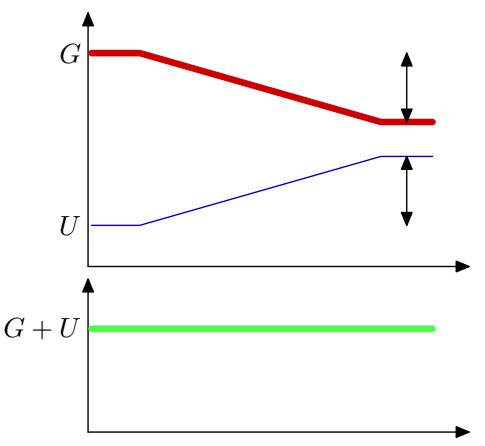

(b)

Figure 1. (a) When one molecule is released from a confined space into a larger space, there is a substantial reduction in chemical free energy. If an idealized phosphate ion, for example, is initially confined to a tiny accessible volume $v_{0} \simeq 1 \AA^{3}$ adjacent to an ADP molecule and later it is free to move around a larger volume, say $v_{1} \simeq 800000 \AA^{3}$, then its entropy has increased by $k \ln \frac{v_{1}}{v_{0}} \simeq 13 k$, and the associated free energy decrease is $13 k T$.

(b) For such a drop in the free energy, $G$, to be efficiently converted into mechanical work, $U$, we believe the falling free energy must somehow be directly coupled to rising work in such a way that the total $G+U$ remains roughly constant. This paper presents an explicit model for this coupling.

phosphate ion is simply 'released' into the cellular solution, then $13 k T$ of free energy is wasted. But $13 k T$ is a similar energy to the work done in the power stroke of myosin under optimal conditions, $15 \mathrm{kT}$ according to [How]. It is far too much to allow to go to waste! Nature must use it somehow.

We heed warnings that it does not make sense to evaluate partial molar entropy changes without specifying whether one is operating at constant volume or pressure $[\mathrm{QH}]$, and that it does not make sense to attribute free energy changes to components of a system except after a full cycle [HE]; but the above arguments still provide strong motivation for considering whether release of ligands could drive conformation changes. Release can also decrease free energy by descent of an electrostatic gradient, by hydration or by other processes, as well as by entropy increases. We focus on entropy increases now, and discuss electrostatic effects later, as we believe both are essential to understand the power stroke of actin-myosin.

How can entropy-increase drive conformation changes? Thermodynamics says that to do so efficiently, it must be done slowly enough to allow the system to remain close to a state of constrained equilibrium at all stages (figure 1b); but what could the mechanism be?

For a particle in a trap, there is a natural way to turn its entropy increase of release into work. The particle exerts a pressure $P$ on the walls of the trap, for example $P=k T / v_{0}$ for an ideal gas molecule, which tries to expand the trap. In the case of a trap of size $v_{0}=1 \AA^{3}$ at temperature $T=300 \mathrm{~K}$, this pressure is enormous: $4 \times 10^{9} \mathrm{~Pa} \approx 40000$ atmospheres! If the trap has a mode of expansion (e.g. a piston) coupled to a conformation change then the pressure can push the piston to provide a force to accomplish the conformation change (figure 2 ). If the opposing force is matched to the pressure such that the expansion is done slowly then the pressure does net work $\int_{v_{0}}^{v_{1}} P(v) \mathrm{d} v$, e.g. $k T \ln \left(v_{1} / v_{0}\right)$ for an ideal gas molecule. If the final volume $v_{1}$ is equal to $1 / \rho$, the work done is $-k T \ln \rho v_{0}$, which is $T$ times the entropy change for the release.

This cartoon neglects the external pressure acting on the system and assumes the particle 


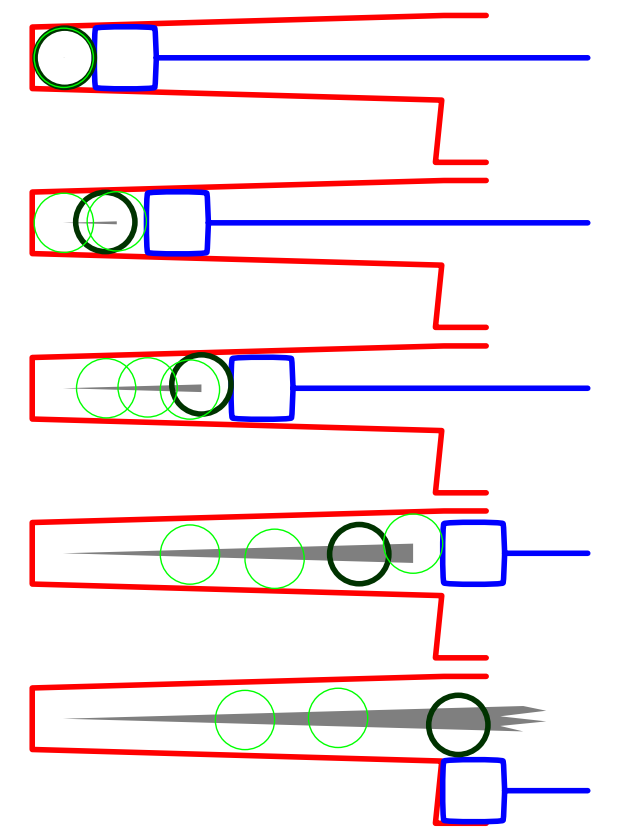

Figure 2. Cartoon of the power stroke of a model engine that converts chemical free energy to work. The circle represents a phosphate ion, trapped in a cleft by a piston (the square) that is coupled to an external load (not shown). The shaded triangular region shows the accessible volume for the centre of mass of the phosphate ion.

The phosphate bounces against the piston; if it succeeds in pushing the piston back, the accessible volume for the phosphate (shown by the shaded triangular region) increases; if the phosphate loses kinetic energy when it bounces against the piston, its kinetic energy will be rapidly restored to its thermal value by subsequent interaction with the surrounding thermal reservoir.

If the piston is coupled to a load given by a potential $U(x)$ (as a function of piston displacement $x)$, such that $\frac{\partial U}{\partial x} \simeq k T \frac{\partial}{\partial x} \ln v(x)$, where $v(x)$ is the volume accessible to the centre of mass of the phosphate, then the work done when the cleft's size has increased from $v\left(x_{0}\right)$ to $v\left(x_{1}\right)$ will be $k T \ln \frac{v\left(x_{1}\right)}{v\left(x_{0}\right)}$. As viewed by the piston, the load should be large (big $\frac{\partial U}{\partial x}$ ) for small $x$ and smaller for large $x$, so appropriate nonlinear gearing would be required to match to a constant load. 
is an ideal gas. The mechanism is not restricted to ideal gases, however. In liquids, the same entropic effect goes by the name "osmotic pressure". Osmotic pressure is exerted on a semipermeable membrane (permeable to the solvent but not the solute particles) when there is a difference in density of solute on the two sides; the pressure reflects the entropy increase that would be achieved by moving the membrane to equalize the densities. Osmotic pressure is usually regarded as an effect of large numbers of solute particles, but osmotic pressures can be defined equally well, in a time-averaged sense, when one or both sides have a small number of solute particles. The pressure due to a single solute ion in volume $v_{0}$ is again given by the ideal gas formula, $P=k T / v_{0}[\mathrm{LL}]$.

When the number of relevant molecules is small on at least one side of a membrane, we call the effect "ergodic pumping". The terminology reflects the fact that the molecules exert a time-averaged force ("ergodic theory" is the study of time-averages for dynamical systems). It seems rare for biochemists to think about forces on conformation changes other than attraction and repulsion, though the idea of pressure forces is used in cell mechanics, e.g. [HK, HMD]. In this paper, the 'membrane' is embodied by a single protein molecule, and the two sides of the membrane are the interior and exterior of that molecule.

The idea that entropy increases can drive biochemical processes is not new [Hill]. For example, the entropy increase of released water is responsible for the binding of hydrophobic residues to each other [L]. Our point is to suggest an apparently overlooked mechanism, ergodic pumping, by which entropy increases can drive conformation changes. In general ergodic pumping can be expected to be responsible for only certain parts of a cycle of conformation change, but we believe that it is likely to be an important ingredient in many biomolecular systems.

First we describe some computer demonstrations of ergodic pumping. Then we review the relevant mathematical theory of kinetics and show how it can lead to ergodic pumping. Next we discuss the idea in some detail in the context of actin-myosin. We hope that this three-pass approach, presenting the same idea from different viewpoints and in different styles, will permit a broad range of readers to appreciate at least some parts of the paper. Finally we speculate on a wide range of other biomolecular systems in which we suspect ergodic pumping plays a significant role, ending by proposing it as a design principle for nanobiotechnology.

The idea was presented at Equadiff in July 2003 [M], a Royal Society of Chemistry Forum in Sept 2003 [MM], a Newton Institute discussion meeting in March 2004, and at the May 2005 Nonlinear Physics conference in honour of Serge Aubry.

\section{Computer demonstrations}

Consider a cycling ATP-hydrolysing system that quickly and near-reversibly converts chemical energy to work. We focus on the key step in this system, before which the chemical free energy is large and the work done is small, and after which the chemical energy has dropped by a large amount (say $13 k T$ ) and the work done has risen by a similar amount. Our idealized system starts this step with a single phosphate particle confined in a tiny volume $v_{0}$. In order to capture the free energy, the phosphate release is directly associated with the doing of work, as depicted in figure 2. 


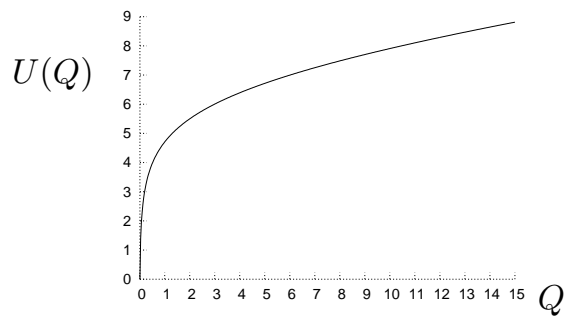

(a)

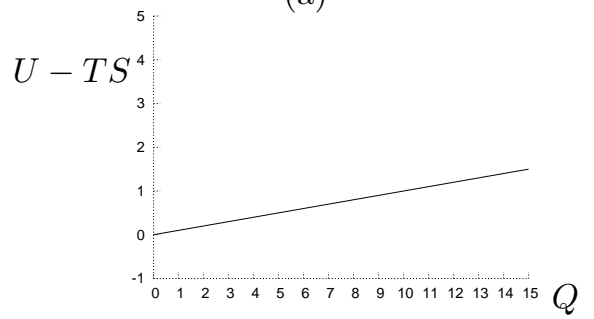

(c)

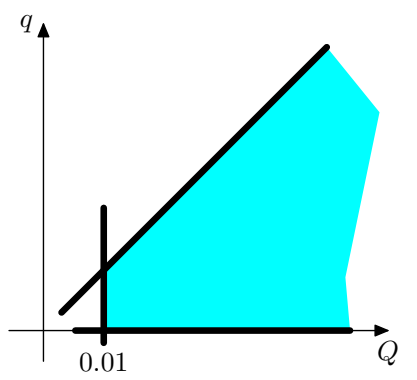

(b)

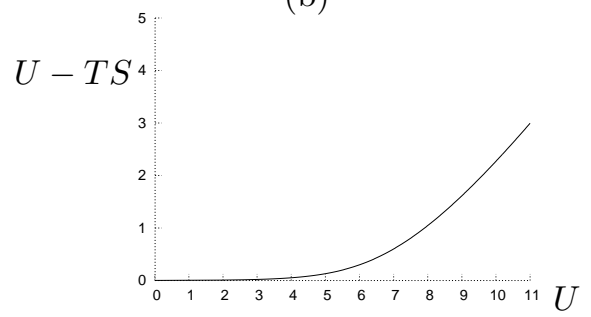

(d)

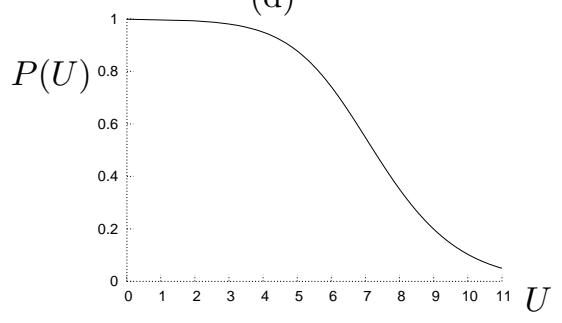

(e)

Figure 3. Parameters of the computer demonstration. (a) Work done versus piston coordinate $Q$, in units of $k T: U(Q)=0.9 \ln \frac{Q}{Q_{0}}+0.1 Q$. (b) The accessible state space for the driver coordinate $q$ and piston coordinate $Q$ is bounded by the constraints $q>0, q<Q$, and $Q>Q_{0}=0.01$. (c) The free energy $U-T S$ varies little with $Q$, by design. (d) The free energy $U-T S$ as a function of the work done, $U$. (f) The equilibrium probability distribution of the work done, $P(U)=\exp (U-T S)$. A range of values of $U$ from 0 to 7 have substantial probability, so the system is expected to pass reversibly between states whose potential energy differs by $6 k T$. 


\subsection{The system}

We simulate a one-dimensional system containing just two particles - the driver (phosphate) and the piston. The state space of the system is shown in figure $3 \mathrm{~b}$. The driver's coordinate $q$ is free to vary between 0 and the piston coordinate $Q$. We couple the piston to an appropriate load $U(Q)$ such that

$$
\frac{\partial U}{\partial Q} \simeq k T \frac{\partial}{\partial Q} \ln v(Q)
$$

where $v(Q)$ is the volume accessible to the centre of mass of the driver, $v(Q)=Q$.

The potential energy of the system is chosen to be (figure 3a):

$$
U(Q)=\ln \frac{Q}{Q_{0}}+0.1 Q
$$

where $Q_{0}=0.01$ is the leftmost permitted coordinate for the piston. We fix the mass of the driver to $m=1$. We choose the piston to have a mass $M(Q)$ depending on its position, specifically

$$
M(Q)=10\left(\frac{\partial U}{\partial Q}\right)^{2}
$$

We chose a position-dependent mass for two reasons. Firstly, in a real biomolecule there is no actual piston - the boundary of the phosphate trap is formed from pieces of protein which move relative to each other and more distant parts in complicated ways; the effective mass is unlikely to be independent of the trap volume. Secondly, if we put a constant-mass piston in a potential like (2), which has very little configuration space with energy within $k T$ of the minimum, thermal fluctuations would take the energy several $k T$ above the minimum even when no driver is present; such spontaneous fluctuations would obscure the effect of ergodic pumping by the driver. For a piston whose effective mass increases sharply near the minimum of the potential, the small configuration-space volume available near the minimum is compensated for by a large momentum-space volume. The specific choice (3) makes the probability distribution for the energy of the piston proportional to

$$
\exp \left[-\left(U-U_{0}\right) / k T\right]
$$

where $U_{0}$ is the minimum possible energy, $U\left(Q_{0}\right)$, so the average potential energy relative to that minimum is $1 k T$. Thus when we introduce the driver, we can attribute increases of potential energy significantly bigger than $k T$ to the presence of the driver.

The system is maintained at temperature $k T=1$ by thermalizations of the driver's velocity whenever it hits the left-hand wall. Whenever the driver hits the wall $q=0$, the driver's velocity $v$ is drawn from the appropriate thermal distribution $\propto|v| \exp \left(-m v^{2} / 2\right)$. [The factor of $|v|$ must be included to give the thermal distribution for particles passing a particular point, in contrast to the thermal distribution for particles at a particular time, which is $\propto \exp \left(-m v^{2} / 2\right)$.] When the piston hits the wall $Q=Q_{0}$, the piston's velocity $V$ is thermalized similarly. Hamiltonian dynamics apply everywhere else. 199 out of every 200 collisions between the piston and driver $(q=Q)$ are elastic (energy and momentum are conserved). On every 200th collision between piston and driver, momentum is conserved, and the relative velocity is thermalized. (This rule was added to avoid infinitely long simulations when the piston and driver chatter against each other.) Between collisions, the Hamiltonian dynamics are simulated using the leapfrog method. Source code may be viewed at http://www.inference.phy.cam.ac.uk/mackay/dynamics/ myosin/c/. 


\section{$2.2 \quad$ Predictions}

We can predict the behaviour of the piston coordinate $Q$ by marginalizing over $q$, whose accessible volume, conditional on $Q$, is $v(Q)=Q$. In energy terms, the driver adds an entropic contribution

$$
T S(Q)=k T \ln \frac{Q}{Q_{0}}
$$

to the energy landscape $U(Q)$ (figure 3c). The predictions for the probability distribution of the work done, $U$, are plotted in figure $3(\mathrm{e})$.

\subsection{Results}

A single simulation of the system is shown in figure 4. At time zero, the driver is at $q=0.005$ and the pocket is almost at its smallest permitted volume: $Q=0.0101$. We set the driver velocity and piston velocity to typical thermal values. Between collisions, the driver coordinate $q$ varies linearly with time, because the potential is independent of $q$.

The characteristic pattern while work is being done (eg, figure 4a, where the piston is moving away from zero) is this: after each collision of piston and driver, the speed of the driver is smaller than it was before; the driver then collides with the wall $q=0$ and its velocity is re-thermalized, leading, typically, to an increase in speed; so the zigzag trajectory of the driver in that period consists of steep upward segments and slightly less steep downward segments. Thus heat is sucked from the surroundings into the driver and turned into work, just as in the isothermal expansion of a heat engine.

This simulation used a simple one-dimensional pocket, rather than the triangular region depicted in figure 2. If we simulate a pocket whose volume grows as $Q^{2}$, as shown in that figure, or, most realistically, as $Q^{3}$, the entropy increases as $2 k \ln Q$ or $3 k \ln Q$, so the force exerted by the driver is two or three times greater.

Our model so far has a defect: to extract all the free energy associated with the release of phosphate into a solution where the concentration is one phosphate per $800000 \AA^{3}$, a tube of size $800000 \AA^{3}$ is required. This is implausibly large. We now describe a fix for this defect that allows the full free energy to be reversibly extracted with a tube whose volume is a thousand times smaller.

\subsection{The charged exit path}

We add positive charges to the mouth of the tube, as shown in figure 5. As we explain below, the energy associated with bringing a negatively-charged phosphate ion close to some positive charges could easily be about $7 k T$, which makes the equilibrium density at the mouth of the tube higher by a factor of $e^{7} \approx 1000$ : one phosphate per $800 \AA^{3}$ instead of one phosphate per $800000 \AA^{3}$. If the volume of the tube is about $800 \AA^{3}$ then the release of phosphate can be a reversible process.

How does this relate to getting the second half of the free energy turned into work? The attraction of phosphate to the positive charge will increase the pressure exerted by the phosphate on the piston as the piston approaches the end of the tube. The potential $U(x)$ should be designed such that this extra force is exploited to obtain extra work.

This model embodies several concrete predictions. We predict that all ATP-hydrolysing motors, if they are efficient, will have positive charges in the vicinity of the phosphate exit- 

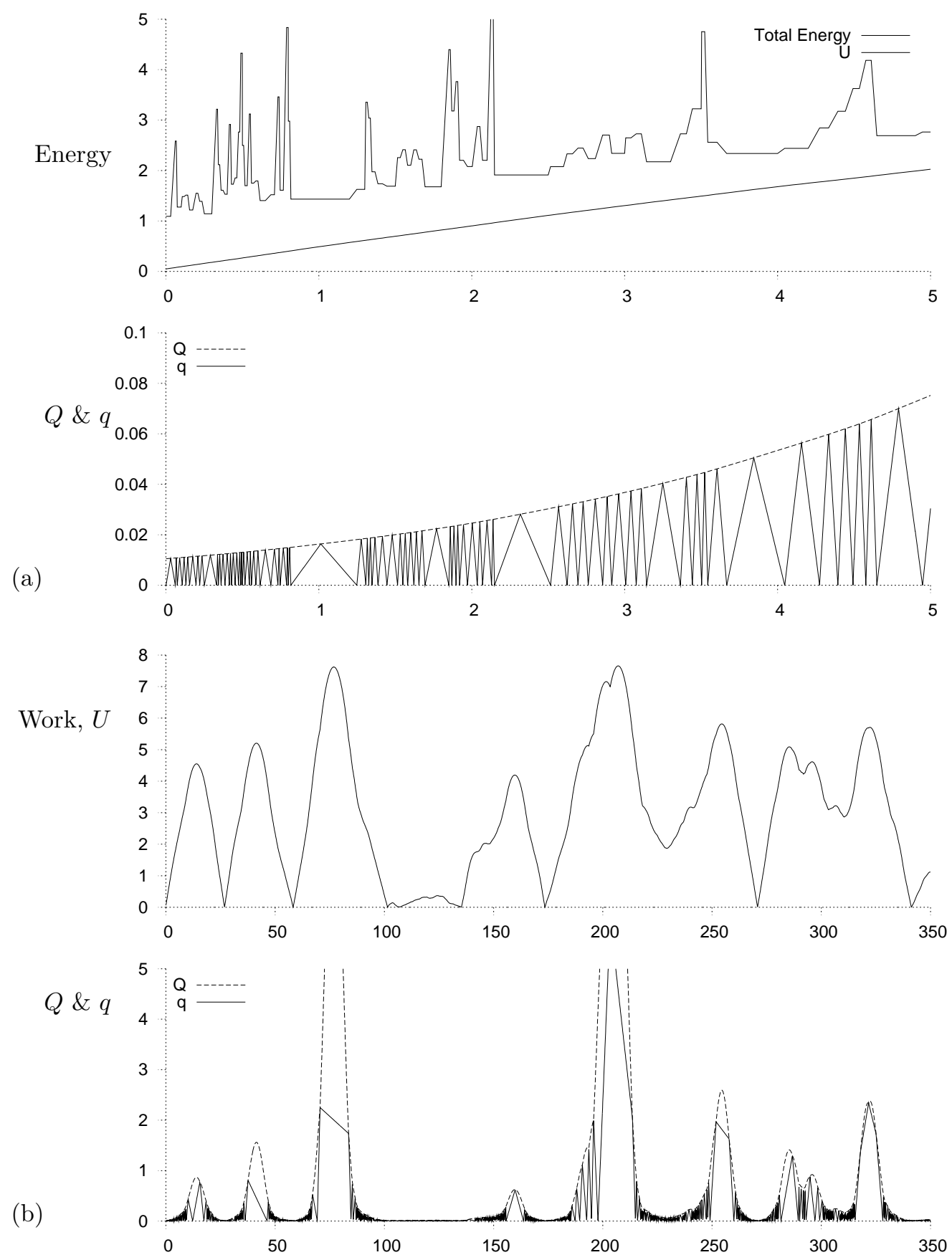

Figure 4. System state and energy as a function of simulation time. (a) The first 5 time units, starting from initial condition $q \simeq 0, Q \simeq 0.01$. After each collision between the driver and the retreating piston, a little energy is transferred from driver to piston. The driver's energy is typically boosted again by the thermalizations when $q=0$. (b) The first 350 time units. As predicted, the work done wanders reversibly between 1 and $7 k T$. 


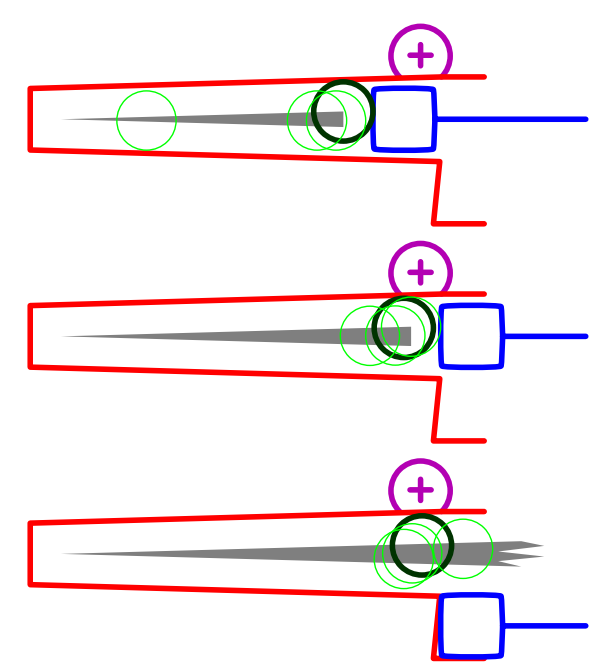

Figure 5. Enhanced model engine with a positive charge near the exit-port for the negatively charged phosphate ion. This charge increases the local equilibrium concentration of phosphate so that phosphate release becomes a reversible step that does not waste free energy. Extra work can be done during the expansion if the external load is increased appropriately to match the increased pressure exerted by the phosphate; the phosphate exerts a higher pressure during the expansion because it is attracted to the positive charge.

port. We predict that the exit-port will have a fairly strong binding affinity for phosphate. And we predict that mutations that reduce the affinity of the exit-port for binding phosphate will lead to a reduction in efficiency of the motor: the motor may still run as fast, but the maximum work it can do will be reduced. (This last prediction may not be obvious if one thinks of phosphate as a reaction product that should simply be expelled so that the motor can continue its cycle; in such a view, surely it would be best for the phosphate not to feel any attraction back to the exit-port, the better to be expelled?)

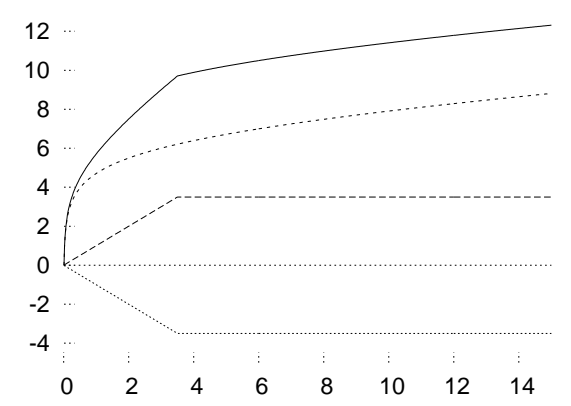

Figure 6. New potentials. From top to bottom: the new potential for the driver, as a function of $Q$; the old potential for the driver, as a function of $Q$; the extra resisting potential for the driver $U_{\text {increment }}^{(2)}(Q)$; and the attractive potential for the driver, $U_{\text {increment }}^{(1)}(q)$, as a function of $q$. All potentials are shown in units of $k T$. 


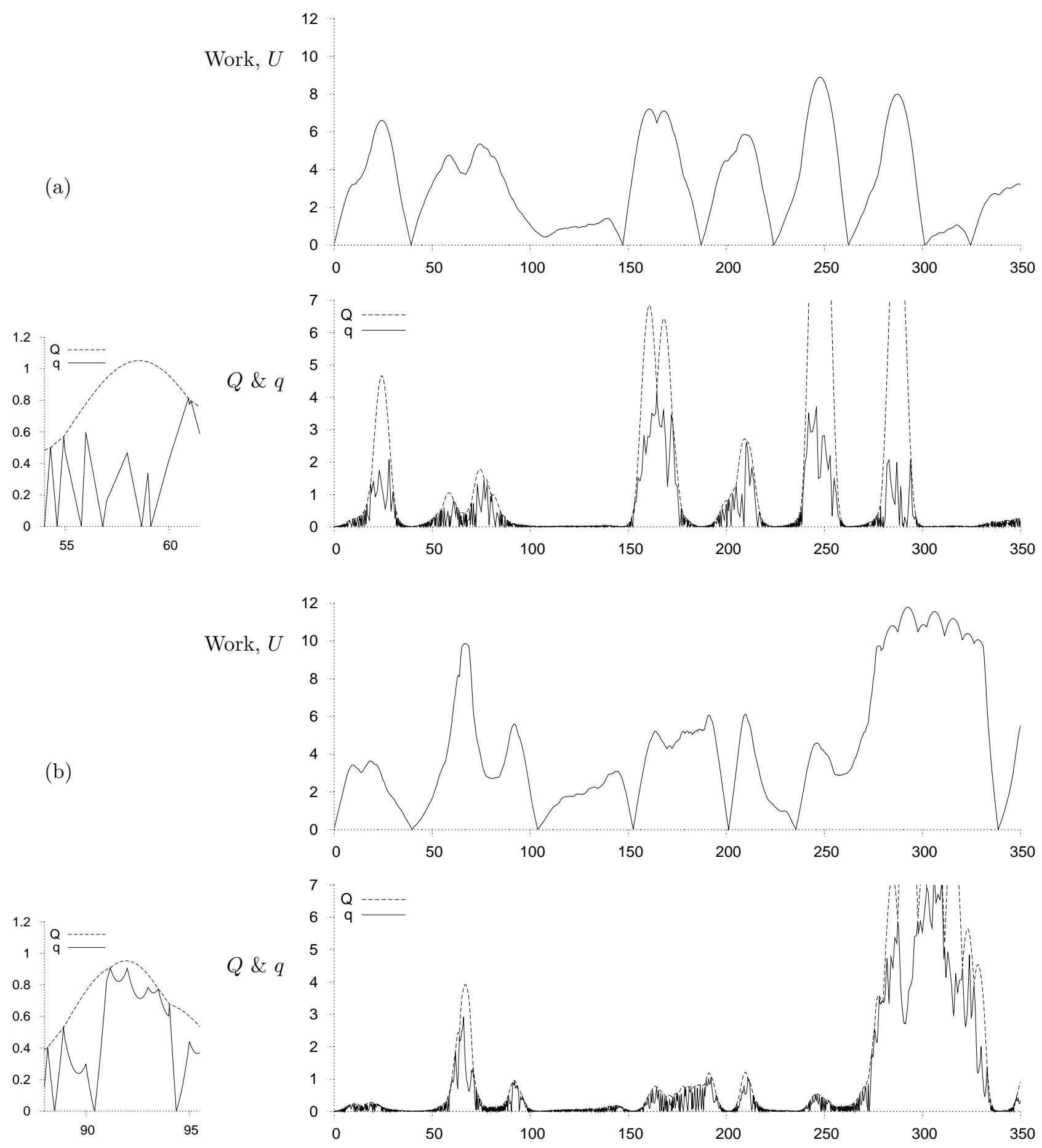

Figure 7. (a) Simulation of a system identical to figure 4's, except that the velocity of the driver is thermalized every 1 time units. (b) Simulation of the system with the charged exit path. The piston and driver potentials are modified as shown in figure 6. In all figures, horizontal axis is simulation time. 


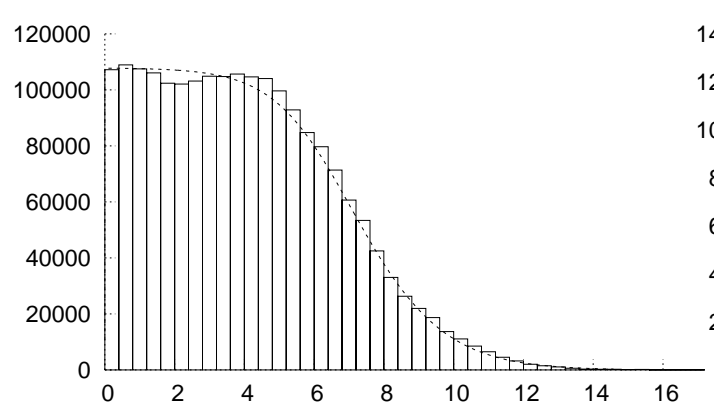

(a)

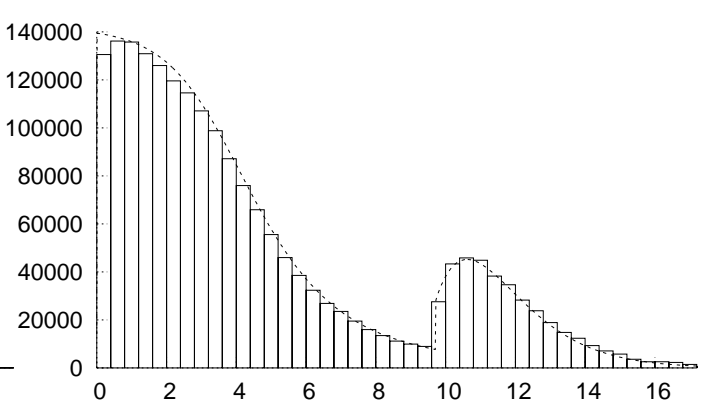

(b)

Figure 8. Predicted (dashed) and actual (solid) histogram of work done, for 2000000 samples from a simulation of duration 200000 time units. In both graphs, the horizontal axis is the work done, $U$, in units of $k T$. (a) Predictions and results for the simulation with frequent thermalizations but without the charged exit-path modification (These predictions were first plotted in figure 3e.) (b) Predictions and results for the charged exit-path simulation.

\subsection{Simulation of the charged exit-path model}

We modify the one-dimensional system by adding an attractive potential for the driver

$$
U_{\text {increment }}^{(1)}(q)=\left\{\begin{array}{cc}
-q & q<3.5 \\
-3.5 & q \geq 3.5
\end{array}\right.
$$

and an additional resisting potential for the piston

$$
U_{\text {increment }}^{(2)}(Q)=\left\{\begin{array}{cc}
Q & Q<3.5 \\
3.5 & Q \geq 3.5
\end{array} .\right.
$$

The new potential for the piston is the top curve in figure 6. If the driver gets past coordinate $q=3.5$, then an extra $3.5 k T$ of work will have been done. In exchange, the released driver will now be in a potential of $-3.5 k T$; escaping from this potential well is difficult, so the released driver will be at equilibrium with an external solution where the driver concentration is smaller by a factor of $e^{3.5}$.

For efficient simulation, we increase the rate of thermalizations of the driver. We introduce additional thermalization events, one per time-unit, at which the velocity of the driver is drawn from the Boltzmann distribution. To clarify the effect of these two modifications to the model, figure 7 shows two simulations, one (7a) with the extra randomization events only, and one (7b) with the charged exit path modification.

In the detail inset in figure 7a, we see that the driver, which in the first simulation moved in straight lines until it encountered wall or piston, now pursues an undirected random walk. The piston, as before, moves along slightly curved trajectories because of the force $\partial U / \partial Q$.

In the detail of figure $7 \mathrm{~b}$, we see that (for $q \in(0,3.5)$ ) the driver follows parabolas directed towards the piston; so the driver tends to be closer to the piston and hit it more frequently than before. The piston moves along more curved trajectories because the force $\partial U / \partial Q$ is now greater by 1 , for $Q \in(0,3.5)$. During the period 270-330, the driver drives the piston up this additional $3.5 \mathrm{kT}$ barrier, and the amount of work that can be reversibly done and undone is increased. 
As a check on these simulations, predicted and actual histograms of the work done are shown in figure 8 . In the final simulation, the system frequently reaches a state in which the work done is $11 k T$.

\section{Isothermal Kinetics}

The possibility of driving conformation changes by ergodic pumping follows from thermodynamics, but thermodynamics is usually formulated for macroscopic quantities so that fluctuations from averages are negligible, whereas we wish to study the behaviour of a single biomolecule or small group of biomolecules subject to their environment. Furthermore, thermodynamics does not predict the rates at which changes will happen, nor quantify what counts as a slow enough change for free energy not to be wasted. So one has to consider microscopic models.

We follow the standard belief [BKO] that slow degrees of freedom of a large system in thermal equilibrium can be regarded as evolving by a Langevin equation with thermal forcing noise and associated damping, a line of thought initiated by Einstein 100 years ago [Ei] and brought into chemistry by Kramers [HTB]. Its foundations will be examined elsewhere, but here we spell out what we believe to be a correct general formulation.

We consider the slow variables in canonically conjugate pairs $Z_{i}=\left(Q_{i}, P_{i}\right)$ (configuration and momentum) for $i=1, \ldots, N$, and denote the whole set of slow variables by $Z$. Reduction to only configuration coordinates $Q$ will be discussed later. We let $\beta=1 / k T$, and denote the free energy given $Z$ by $F(Z)$. ( $F$ also depends on $T$.) In most treatments $F$ is regarded as a function of only $Q$, not $P$, and it is then called the "potential of mean force" or the "configurational energy landscape", but it can be defined equally well for $Z=(Q, P)$. We denote by an overbar averages with respect to the conditional distribution of the canonical ensemble on a prescribed value of $Z$. For the present exposition we work at constant volume, but the discussion could be adapted to constant pressure. For subsystems that catalyse a chemical reaction that is maintained out of equilibrium, it is appropriate to consider $Z$ to include information about the numbers of molecules on each side of the reaction. (Mathematically we would say we are working in a covering space over the slow variables.) So following a cycle in $Z$ changes $F(Z)$ by the free energy change for the reaction.

Then $\dot{\bar{Z}}=J \nabla F(Z)$, where $J=\left[\begin{array}{cc}0 & I \\ -I & 0\end{array}\right]$ and $\nabla$ is the gradient with respect to $Z$. We denote the deviations of $\dot{Z}$ from this average by $\zeta$, and let $D_{i j}(Z)=\frac{1}{2} \int_{0}^{\infty} \overline{\zeta_{i}(t) \zeta_{j}(0)} \mathrm{d} t$ (assuming the integral converges), where the time evolution is that of the system constrained to constant $Z$. Then $Z(t)$ is believed to evolve by a Langevin equation

$$
\dot{Z}_{i}=\left(J_{i j}-\beta D_{i j}(Z)\right) \nabla_{j} F(Z)+\gamma_{i j}(Z) \dot{W}_{j}(t)
$$

where $W_{j}$ are independent Wiener processes, $\gamma(Z)$ is a matrix with $\gamma \gamma^{T}=D+D^{T}$ (superscript $T$ denotes transpose), summation over repeated indices is assumed, and the equation is to be interpreted in the backwards Ito sense, i.e., as the limit as $\tau \rightarrow 0$ of the discrete-time process

$$
Z_{i}((n+1) \tau)-Z_{i}(n \tau)=\tau\left(J_{i j}-\beta D_{i j}\right) \nabla_{j} F+\gamma_{i j}(Z((n+1) \tau)) w_{j}(n)
$$

with $w_{j}(n)$ independent random steps of zero mean and variance $\tau$. ("Backwards" means that the coefficient of $\dot{W}$ is evaluated at the end of each step, rather than the beginning). Although we are neither aware of a rigorous derivation (for a review, see $[\mathrm{GKS}]$ ), nor of anyone pointing out 
before that one should use the backward Ito convention, this (or special cases or extensions to time-dependent damping and time-correlated noise) is what most biochemists assume, possibly in different notation. In particular, it follows that the probability density $\rho$ for $Z$, with respect to Liouville measure $d Z$ for the slow system, evolves according to a Fokker-Planck equation

$$
\frac{\partial \rho}{\partial t}=-\nabla_{i}\left(\left[\left(J_{i j}-\beta D_{i j}\right) \nabla_{j} F\right] \rho-D_{i j} \nabla_{j} \rho\right) \text {, for } t>0 .
$$

In the special case that the Hamiltonian has the simple form

$$
H(Q, P, z)=\frac{1}{2} P^{T} M^{-1} P+h(Q, z)
$$

for some (possibly $Q$-dependent) positive-definite symmetric matrix $M$, where $z$ denotes all the remaining variables, $F(Q, P)$ takes the form $\frac{1}{2} P^{T} M^{-1} P+G(Q)$, with $G$ the usual free energy function of configuration coordinates $Q$ (though still at constant volume rather than constant pressure, so this is Helmholtz rather than Gibbs free energy). Then $\dot{Q}=M^{-1} P$ and $\zeta$ has only $P$-components, so $D$ and $\gamma$ reduce to matrices relating only components of $P$ and depending only on $Q$. The stochastic equation reduces to

$$
M_{i j} \ddot{Q}_{j}=-\nabla_{i} G(Q)-\frac{1}{2} \dot{Q}_{k} \nabla_{k} M_{i j} \dot{Q}_{j}-\beta D_{i j} \dot{Q}_{j}+\gamma_{i j} \dot{W}_{j}(t) .
$$

If the slowest damping rate $\beta\left\|M D^{-1}\right\|^{-1}$ is fast compared to the maximal rate of change of $M, D$ and $G$ along solutions (due to their $Q$-dependence), then the second derivative and $\nabla M$ terms can be eliminated, to a good approximation, resulting in a stochastic equation that is more commonly used than (8):

$$
\beta D_{i j} \dot{Q}_{j}=-\nabla_{i} G(Q)+\gamma_{i j} \dot{W}_{j}
$$

which can alternatively be written as

$$
\dot{Q}=-k T D^{-1} \nabla G+2 k T \gamma^{-T} \dot{W}
$$

where $\gamma^{-T}$ denotes the inverse of $\gamma^{T}$. The associated equation for the evolution of the probability density $\rho$ for $Q$ with respect to volume $d Q$ in slow configuration space is the Smoluchowski equation:

$$
\frac{\partial \rho}{\partial t}=\operatorname{div}\left(\left(k T D^{-1} \nabla G\right) \rho+k^{2} T^{2} D^{-1} \nabla \rho\right),
$$

which may be more familiar (e.g. [MB]) than (10).

We prefer to stick with the general form (8), however, because it allows the kinetic energy to contain cross-terms involving both slow and fast momenta, which is generically the case; for example, if one component of $z$ represents the state of a side chain relative to the conformation change variables $Z$, then its contribution to the kinetic energy will include terms of the form $(P+p)^{2}$.

To compute $F$ and $D$ ab initio is fairly hopeless, but in small enough systems they can be estimated from molecular dynamics simulations where constraint forces are added to keep the slow variables fixed [BEL, MB]. 
Now consider what (8) tells us about the dynamics of conformation changes. Using the backwards Ito version of stochastic calculus (which we have not found written anywhere; see $[\mathrm{O}]$ for the forward Ito calculus), the rate of change of $F(Z)$ along a solution is

$$
\frac{d}{d t} F(Z)=\nabla F^{T}(J-\beta D) \nabla F+\nabla F^{T} \gamma \dot{W}-D_{i j} \nabla_{i} \nabla_{j} F
$$

Since $J$ is antisymmetric, the contribution $\nabla F^{T} J \nabla F$ is zero, and since $D$ is non-negative, the contribution $-\beta \nabla F^{T} D \nabla F$ is non-positive. The final term $-D_{i j} \nabla_{i} \nabla_{j} F$ is non-positive near a local minimum of $F$, but the noise term has non-zero mean $\nabla\left(\nabla F^{T} D \nabla F\right)$, which is non-negative near any critical point. Thus $F(Z(t))$ typically decreases as $t$ increases, until close enough to a local minimum of $F$ that the remaining terms may cause it to settle to thermal equilibrium. So $Z(t)$ drifts generally downhill in the free energy landscape (albeit with a dominant component along level sets of $F$ because of the $J \nabla F$ term in (8), though this is eliminated if the system is reduced to first order in $Q$, as in (14)), until it reaches a neighbourhood of a local minimum (transitions over barriers to other local minima may take place on a longer timescale). Under "hypoellipticity" assumptions on $D,(8)$ has a globally attracting equilibrium density for $Z$, namely $e^{-\beta F(Z)}$, up to normalisation.

It is convenient to think of many conformation changes (e.g. actin-myosin) as transferring free energy from an internal system to an external one, albeit with some inevitable loss. For example, actin-myosin can transfer free energy from muscle cells into lifting weights. Even for conformation changes that do not do such obvious external work, this point of view is useful, e.g. kinesins turn free energy of hydrolysis of ATP into motion of cargo, which does work against viscous forces; even though the viscous forces are in principle just the result of the same Langevin equation applied to the cargo, it is convenient to regard them as external.

It is simplest to think about the case where the external forces are conservative, so the system can be represented by a Hamiltonian of the form $H_{\text {ext }}(Z)+H_{\text {int }}(Z, z)$, where $H_{\text {ext }}$ is considered as "external energy". Then

$$
F(Z)=H_{\text {ext }}(Z)+F_{\text {int }}(Z)
$$

with $F_{\text {int }}$ being the internal free energy. Suppose $Z$ is started in a state that is not a minimum of $F_{\text {int }}$. To obtain a significant increase in $H_{\text {ext }}$, we had better have at least a corresponding and nearly monotone decrease in $F_{\text {int }}$. To convert a large fraction of the decrease in $F_{\text {int }}$ to an increase in $H_{\text {ext }}$, we had better have the dissipation rate $\beta \nabla_{i} F D_{i j} \nabla_{j} F$ small along the path. An internal free energy decrease can be converted to work with high efficiency if and only if the function $H_{\text {ext }}$ matches $F_{\text {int }}$ well, in the sense that there is a probable path for (8) with only small downhill slope for $F=F_{\text {int }}+H_{\text {ext }}$.

This point of view is fundamentally different from that of [Hill], who considers a partition of the state space into a discrete set of "states", which are assumed to be in internal equilibrium even if they possess parameters like conformation state variables, which would imply that free energy is not dissipated at all within a state, ignoring the well known phenomenon of viscous dissipation. Hill's view also makes it impossible to account for the fact that entry into a state like the power stroke state for actin-myosin has to occur in some window of the state variables and exit from a different window, or to account for the time spent in achieving change of the state variables in between. Also Hill's view makes it complicated to include continuously progressive binding - one has to introduce a sequence of states which may not really have clear boundaries between them. 
Our point of view is also different from that of many "thermal ratchet" models, which are popular among physicists $[R]$. They often invoke an artificial external switching between chemical states and study the resulting motion of a biomotor along a (linear or circular) track. Our criticisms of such models are firstly that in reality the switching between chemical states is a result of the chemical process, not an externally imposed cycle. Secondly, thermal ratchet models tend to employ significantly irreversible steps which make it difficult to explain the high free-energy-to-work conversion efficiencies achieved by many biological motors. A synthesis of the ideas, however, is presented in $[\mathrm{BKO}]$.

\section{Actin-Myosin}

Now we make our main application. Actin and myosin are the principal players in the generation of force and movement by muscle. Actin polymers form long filaments (the "thin" fibres). The myosin tails matt together in long filaments parallel to these (the "thick" fibres). After a myosin head attaches to a binding site on actin, it undergoes a conformation change pulling the thick fibre relative to the thin fibre. If this "power stroke" goes to completion, or after some time, the head detaches and changes back to its original conformation, ready to go round the cycle again. The cycle is driven by hydrolysis of ATP (adenosine triphosphate) to ADP $+\mathrm{P}$ (ADP $=$ adenosine diphosphate, $\mathrm{P}=$ phosphate ion), a reaction that is maintained far from equilibrium in most cells. We refer to the conformation of myosin before the power stroke as "extended" and after as "hooked" ([GH] call these states "up" and "down" respectively).

At a biochemical level, the basic cycle is believed to be

DETACHED

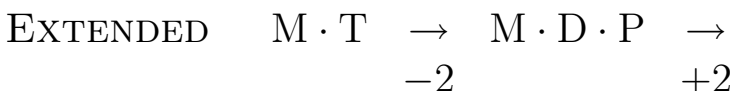

HOOKED
AtTACHED

$\mathrm{A} \cdot \mathrm{M} \cdot \mathrm{D} \cdot \mathrm{P}$

$$
\downarrow-12
$$

$\mathrm{A} \cdot \mathrm{M} \cdot \mathrm{D}$

$\downarrow-3$

$$
\mathrm{A} \cdot \mathrm{M} \cdot \mathrm{T} \stackrel{-2}{\leftarrow} \quad \mathrm{A} \cdot \mathrm{M}
$$

where $\mathrm{M}=$ myosin, $\mathrm{A}=$ actin, $\mathrm{T}=\mathrm{MgATP}, \mathrm{D}=\mathrm{MgADP}$ (ATP and ADP carry a magnesium ion with them most of the time), and the numbers indicate approximate free energy changes in units of $k T$ per molecule (for rabbit skeletal muscle under no tension at physiological conditions, adapted from [How]). On the left side the myosin is detached from the actin, whereas on the right it is attached (though with varying degrees of binding which are not shown). The free energy drops by $25 \mathrm{kT}$ per cycle. For comparison, the work done by muscle under optimal conditions is estimated at a maximum of $15 k T$ per cycle. This can be presumed to come mainly from the right-hand part of the cycle where the myosin is attached to the actin and changes conformation from extended to hooked (though the $8 k T$ decrease from AMT to MT also looks too good to waste and merits future consideration).

It should be noted that the above cycle is not universally accepted, and the details may depend on the type of myosin (of which there are 18 main classes [BPC]). For example, [SH] indicates $\mathrm{Mg}$ release as a separate step between release of $\mathrm{P}$ and $\mathrm{ADP}$ for myosin $\mathrm{V}$ (and shows 
a slightly different cycle in [HoS]). The most serious issue under dispute is whether the AMDP to AMD step has an intermediate extended AMD* state. Some authors believe P is pushed out, creating a "highly strained AMD* state" [How] which then undergoes the major conformation change (see also $[\mathrm{AC}, \mathrm{SH}, \mathrm{Re}+]$ ), but others are sure that $\mathrm{P}$ release occurs after the main working stroke (e.g. [GH, SS, TSG]). We will work with the second hypothesis, though we will indicate later how we could incorporate the first if it turns out to be the case for some myosins. Another detail, which will not be relevant for us, is that it appears the MT to MDP transition proceeds via an intermediate $\mathrm{MT}^{*}$ state $[\mathrm{MC}+]$.

How is the free energy decrease in the power stroke turned into work? We propose that it is principally by ergodic pumping. Here is our proposed story, based on the above principles, the experimental literature, and structures determined by crystallography, studied with the aid of Protein Explorer. Details might be wrong, but we believe that something like this story must be true.

Let us first dispose of a common misconception. Contrary to the cartoon of [VM], hydrolysis of ATP in the ATP-binding pocket of myosin does not liberate a flash of energy which is then stored in some unidentified place such as vibrations or springs and released later as work in the power stroke. In fact, the hydrolysis of ATP in the myosin pocket is endothermic $(\Delta H=$ $+27 k T$ ) and reversible with an equilibrium constant of about 10 [MHG] (so $\Delta G=-2.3 k T$ ). Thus, an amount $T \Delta S=+29 k T$ of heat is sucked in from the environment on hydrolysis. Part of the entropy increase this represents could be due to tumbling of the $\mathrm{P}$ in the pocket, but there are myosin conformation changes too $[\mathrm{MC}+]$ which could account for the rest.

Thus, to begin our story, before attachment to actin, the ATP in the ATP-binding pocket of the myosin has already hydrolysed. The resulting $\mathrm{P}$ is confined, however, to a small volume at the inner end of the binding pocket. We claim it exerts a pressure on this part of the pocket, attempting to expand it. It is not until actin begins to bind to the myosin, however, that the expansion is able to start. We presume the actin binding weakens the tightness of the pocket (compatible with suggestions of [GH]), perhaps initiates the expansion, and in particular allows the $\mathrm{P}$ to detach from the magnesium ion which has still been holding it to some extent. The $\mathrm{P}$ expands the inner end of the pocket (the "cleft" of $[\mathrm{F}+]$ ), driving the beginning of the believed rotation of one domain relative to another and permitting strengthening of the actin binding (which contributes further free energy). The trap is permeable to water but not to $\mathrm{P}$, so water enters as it expands. At the same time that the $\mathrm{P}$ expands its trap, the resulting expanding "bubble" made by the $\mathrm{P}$ moves away from its original location, initially along the tunnel, since it is almost universally agreed that $\mathrm{P}$ cannot leave past the ADP. There is no consensus, however, about where the $\mathrm{P}$ finally leaves myosin. As we will explain below, we believe it is essential that it leave via a surface region of significant positive charge. Thus, as one candidate for the final location of the bubble, we propose the region between the switch-2 helix, switch-2 loop and the SH1-helix (shown in figure $6 \mathrm{~b}$ of $[\mathrm{GH}]$ ). This region is also plausible because it is known to change shape significantly during the power stroke. The end of the main phase of the power stroke comes with the $\mathrm{P}$ expanding its available volume to about $800 \AA^{3}$, and we propose that this expansion drives the conformation from extended to hooked. The changes produced by the combination of $\mathrm{P}$ pressure and actin binding also drive detachment of the ADP from its binding site (possibly via release of the $\mathrm{Mg}^{2+}$ which probably also does work by ergodic pumping) and it begins to exert a pressure in its pocket. In some myosins it might be that it is only this ADP pressure which drives the main conformation change, allowing for $\mathrm{P}$ release before the power stroke, as some authors claim. 
This would not be the end of the available free energy from the $\mathrm{P}$, however, since the volume available in the cellular fluid is about $800000 \AA^{3}$ per $\mathrm{P}$, one thousand times larger than the final volume proposed above. It is unrealistic to expect an expansion chamber of this size in a biomolecule. So without further ingredients, ergodic pumping could convert to work at most about half of the free energy corresponding to entropy increase associated with phosphate release. We propose that the system extracts the remaining free energy of release of $\mathrm{P}$ by electrostatics, as sketched in section 2.4.

We now fill in the quantitative details of the 'charged exit path' model that we introduced there. The $\mathrm{P}$ carries a charge of between -1 and -2 . The believed in-line hydrolysis reaction produces $\mathrm{HPO}_{4}^{2-}+\mathrm{H}^{+}$[How], but $p K_{a}$ for the $\mathrm{H}_{2} \mathrm{PO}_{4}^{-}$to $\mathrm{HPO}_{4}^{2-}$ transition is about 6.8 and the hydrolysis is observed to release $0.4 \mathrm{H}^{+}$[MHG], so perhaps $60 \%$ of the $2-$ form is converted to $1-$. Outside the myosin, the $\mathrm{pH}$ is about 7.2 , so we would expect $75 \%$ of the $\mathrm{P}$ to end up as $2-$ (though [NFG] suggest $P$ is released as $1-$ ). As the proposed pocket expands to $800 \AA^{3}$, we propose that a mouth opens up, carrying positively charged residues (possibly these are the highly conserved arginines at locations 677, 686, 689 and 695 in Dictyostelium cytoplasmic myosin II; see table 1 of [GH]). Now the equilibrium density of an ion of charge $-c$ with external concentration $C$ is enhanced in a positive potential $\phi$ by a Boltzmann factor of $e^{\beta c \phi}$. Thus $\phi$ of the order of $0.11 \mathrm{~V}$ averaged over the pocket would suffice to match the external concentration (since the thermal energy at $300 \mathrm{~K}$ is $k T \approx 0.026 \mathrm{eV}$ ). (The screening length associated with water's dipoles is about $5 \mathrm{~nm}$, so one can expect charged residues to influence the potential over a volume of up to about $500 \mathrm{~nm}^{3}$.) So in addition to the work done by expansion, the $\mathrm{P}$ would also "descend" an electrostatic gradient, turning the resulting energy into further work on the expansion. The $\mathrm{P}$ starts in an environment that looks neutral or negatively charged, because apart from the $\mathrm{Mg}^{2+}$, the two phosphate groups of ADP carry a total of about $3-$, and there are several negative residues around the $\mathrm{P}$.

\subsection{What happens next?}

Once the $\mathrm{P}$ is in equilibrium with the outside, it is free to leave, and can do no more work. At this stage some sort of energetic obstacle is required to prevent the conformation change from reversing; one possible mechanism would exploit the charge of the phosphate again: the pocket might have some positive charges inside, placed such that in the absence of phosphate there is a substantial energy barrier against the pocket's collapse; the phosphate, if present, neutralizes these charges and lowers the barrier.

When the phosphate is near release, we propose that the ADP takes over as the main force exertor and the $\mathrm{P}$ expansion pocket decouples from the lever arm movement. Detachment of the ADP having been initiated by the $\mathrm{P}$ expansion, ADP exerts force by ergodic pumping, with rotational and flexural as well as translational entropy increases contributing to the force. Expansion of the ADP binding pocket provides a little more movement (observed in some slow myosins; see references on p. 717 of $[\mathrm{GH}]$ ), before the ADP in turn reaches a positively-charged pocket whose size is such that the ADP is close to equilibrium with its concentration outside; the ADP can then leave near-reversibly. The positive charges for the ADP exit are clear, namely the lysines and arginines around the mouth of the ATP binding pocket (e.g. residues 130, 131, 190, 191, 265, 435, 445 and 587 in Dictyostelium cytoplasmic myosin II). The large number of positive charges is required to equilibrate with $20 \mu M$ ADP outside $\left(80000 \mathrm{~nm}^{3}\right.$ per ADP). In the process of the ADP pocket expansion, the myosin becomes strongly bound to actin $[\mathrm{H}+]$, 
thus preventing recollapse of the ADP binding pocket. This is now the rigor state.

To complete the cycle, myosin has to wait for an ATP to turn up and get attracted into the binding pocket; as the ATP binds, it weakens the binding of the myosin to actin, and the myosin floats off the actin. Estimates suggest a significant free energy decrease during this step, which is too large to waste, so we suspect that during the unbinding from actin some further work is done. It would be interesting to understand the mechanics of the release of myosin. A different type of ergodic pumping might occur in which partial release of the myosin allows parts to move more vigorously and hence exert a net repulsive force. Completion of the binding of ATP closes the ATP-binding pocket, reverting the myosin to its extended state. Once the pocket is closed, the appropriate residues are in place to catalyse hydrolysis of the ATP.

\subsection{Evidence}

We have incorporated much experimental evidence into the above proposal. We defer a detailed comparison with experiments to a paper aimed directly at biologists, but list here some further experimental results we must take into account. Increase of $[\mathrm{P}]$ decreases isometric tension and has no effect on the speed of shortening $[\mathrm{CP}]$. It also reduces stiffness and increases the rate of attainment of final tension $[\mathrm{TSG}]$. Increase of the ratio $[\mathrm{ADP}] /[\mathrm{ATP}]$ increases isometric force and decreases speed [CP, FGR, NFG]. Addition of osmotic equivalents of P, e.g. phenyl phosphate, arsenate or vanadate, reduces maximum tension [NFG]. $10 \mathrm{MPa}$ external pressure reduces tension by $10 \%$ [FGR]. pH also has effects [MHG, BLBT] (the power stroke releases about 0.4 of a proton). There are further results on $\mathrm{P}$ release and force generation in [HoS, SS]. There are various attempts to ascertain the route by which $\mathrm{P}$ exits myosin, e.g. [YLR, Re+] give two conflicting proposals (neither of which corresponds to our tentative one). There are several studies of natural or artificially induced mutations of myosins $[\mathrm{GH}, \mathrm{Z}+]$.

Added to the positive evidence for our proposal is the failure of anyone else to propose a mechanism (in the sense of how free energy is turned into work). It is generally believed that binding of the ATP to myosin winds up a "spring" in myosin whose energy is released on binding to actin [HoS]. But where is the spring? We would say the energy source is the pressure of the confined $\mathrm{P}$.

Finally, we propose some tests of the idea.

\section{Genetic modifications}

One could modify residues that are crucial for our explanation. For example, if one made extra space at the $\gamma$-phosphate end of the ATP binding pocket, in a way that still leads to hydrolysis, we would predict that this change would waste a significant amount of entropy of expansion of available volume for the $\mathrm{P}$ and hence reduce the work that can be done.

One could remove some of the positive charges at the proposed exit route for the phosphate. We predict that the maximum work extractable would decrease (the E683K mutation studied in $[\mathrm{Z}+]$ is relevant to this region, though it adds a positive charge rather than removing one).

\section{Solute effects}

We predict that the addition of small negatively-charged solutes to the solution might shield the positive charges at the exit route, reducing the work that can be done. 
Adding large osmotically-active molecules to the surrounding solution is predicted to reduce the force exerted, even if those molecules have no relationship to the molecules involved in the ATP reaction: if the external concentration of osmotically-active molecules is increased, the expansion of the phosphate's pocket will be opposed by a larger osmotic pressure.

\section{$5 \quad$ Other speculative applications}

\subsection{Tubulin motors}

A similar story might be expected for ATP-driven biomotors on tubulin tracks, like kinesins and dyneins, but the phasing of their mechanical and chemical cycles is believed to be somewhat different from actin-myosin [How]. So instead of ergodic pumping occurring in the power stroke, we believe it occurs in a recovery phase. Nonetheless, it does work in just the same way, and requires the same positive charge exit.

\section{$5.2 \quad \mathrm{~F}_{1}$ ATP-ase}

$\mathrm{F}_{1}$ ATP-ase is the part of ATP-synthase that in "normal" operation synthesises ATP from ADP and $\mathrm{P}[\mathrm{Bo}]$. But if it is detached from the $\mathrm{F}_{0}$ proton motor, or the proton-motive force for the $\mathrm{F}_{0}$ motor is insufficient, then $\mathrm{F}_{1}$ ATP-ase hydrolyses ATP and rotates in the opposite direction (pumping protons against the proton-motive force in the second case). $\mathrm{F}_{1}$ ATP-ase is believed to be well explained by Boyer's binding change mechanism. But we believe this explanation is incomplete, since it does not describe how the large entropy increase of the escaping phosphate is used. ATP-synthase is freely reversible and makes negligible losses, so we believe ergodic pumping must be involved.

\subsection{Some ion pumps}

There are several ATP-driven ion pumps, for example 3Na/2K ATP-ase, sarcoplasmic Ca ATPase, and the $\mathrm{H} / \mathrm{K}$ exchanger in the stomach wall [BTS]. Phosphate release must play a role in their function.

\subsection{DNA and RNA motors}

There are several types of ATP-driven motors which use DNA or RNA as their "track" rather than actin or tubulin. In particular, DNA helicase moves along double-stranded DNA to separate the strands: release of ADP + P opens a cleft between two subdomains of the helicase, thus advancing the separation by one base pair. As another example, to terminate RNA transcription, when $\rho$-protein detects a region of RNA rich in $\mathrm{C}$ and poor in $\mathrm{G}$ it attaches to the RNA and pulls itself along towards the RNA polymerase by ATP hydrolysis and causes the RNA polymerase to release its product. As a third example, the bacteriophage $\Phi 29$ has an ATP-powered motor for packing its DNA into its capsid; it is immensely powerful [S+]. Ergodic pumping by $\mathrm{P}+$ ADP must be a major driving force in all three of these motors. 


\subsection{Protein translocators}

There are ATP-driven motors which translocate proteins across membranes, e.g. SecA [W+] and Hsp-70 [El]. Again, ergodic pumping is likely to be used to turn the entropy of release of phosphate into work.

\subsection{Proton and Sodium motors}

Ergodic pumping could also happen with drivers other than phosphate ions. For example, consider torque-producing proton motors like the $\mathrm{F}_{0}$ of ATP-synthase and many bacterial flagellar motors $[\mathrm{Be}]$. These motors are driven by transfer of protons across a membrane from a low $\mathrm{pH}$ (high proton concentration) to a high one. Thus the final step is release of a proton into a low concentration environment. To make use of the resulting entropy increase, we believe the motor must use ergodic pumping (combined with the charged exit path trick, in this case negative charges).

Other bacterial flagellar motors are driven by a flow of $\mathrm{Na}^{+}$ions through a membrane across which a difference in $\mathrm{Na}^{+}$concentration is maintained by pumps. Release of the $\mathrm{Na}^{+}$implies ergodic pumping if the entropy increase is not to be wasted.

\subsection{Others}

Some other biomolecular systems where ergodic pumping could contribute to driving conformation changes are chaperonins [MSXK], G-proteins [Va, NMW] and cytoskeletal polymerisation (actin and tubulin) [How].

\subsection{Nanobiotechnology}

In addition to contributing to understanding conformation changes in natural biomolecules, ergodic pumping has potential as a design principle for nanobiotechnology. It might be possible to design artificial motors, switches and other devices performing conformation changes, driven at least in part by ergodic pumping. Perhaps simplest is to hijack the ATP production in natural cells and design a new device to use it, perhaps also making use of a natural ATP binding pocket, but modifying the use to which the expansion by the phosphate ion is put. There is the potential, however, to design and use entirely different fuels, binding sites, and expansion chambers.

It is worth noting a quantum-mechanical limitation, which nature seems to have recognised. At temperature $T$, a particle of mass $m$ has a thermal length $a=\frac{\hbar}{\sqrt{m k T}}$, where $\hbar$ is Planck's constant divided by $2 \pi$; the particle's position cannot be pinned down more precisely than this (Heisenberg's uncertainty principle says that, for each coordinate, $\Delta x \Delta p \geq \hbar / 2$; and $\Delta p=\sqrt{m k T}$ in thermal equilibrium). So there is a smallest feasible volume from which ergodic pumping can start, of order $(\sqrt{3} a)^{3}$ (the factor of $\sqrt{3}$ is for the case of a cubic trap). For example, for a phosphate ion at $T=300 \mathrm{~K}$, taking $m=150$ atomic units (rather than just the bare 97 or 98 , to allow for the fact that it carries along some water molecules), $\sqrt{3} a \approx 0.07 \AA$. 


\section{Conclusion}

We have proposed a mechanism to convert the entropy increase on release of a ligand into useful work, by letting it expand a pocket until it equilibrates with conditions outside. We have described a computer demonstration of the phenomenon. We have proposed applications to a range of conformation changes, in particular in myosin. We propose ergodic pumping as a design principle in nanobiotechnology.

\section{Acknowledgements}

RSM's thoughts on the mechanism of myosin were initiated by a stimulating proposal of Serge Aubry around 1998, that energy transfer during conformation changes in myosin might be mediated by "discrete breathers" (localised anharmonic oscillations). After hearing a seminar on the functioning of myosin by Mike Geeves in May 2002, however, he became dubious about the discrete breather proposal and instead hit on the idea that release of phosphate must play a major role, and teamed up with DJCM in early Spring 2003 to develop the proposal. We are grateful to Mike Geeves for listening to the ideas and for useful comments. We also thank other participants of the Royal Society of Chemistry workshop at UMIST in September 2003 and a two day Newton Institute discussion in March 2004 for their comments.

\section{References}

[AC] Amos LA, Cross RA, Structure and dynamics of molecular motors, Curr Opin Struct Biol 7 (1997) 239-246.

[BLBT] Baker JE, La Conte LEW, Brust-Mascher I, Thomas DD, Mechanochemical coupling in spin-labelled, active, isometric muscle, Biophys J 77 (1999) 2657-64.

[BEL] Bemporad D, Essex JW, Luttmann C, Permeation of small molecules through a lipid bilayer: a computer simulation study, J Phys Chem B 108 (2004) 4875-84.

[BTS] Berg JM, Tymoczko JL, Stryer L, Biochemistry (Freeman, 2002).

[BPC] Berg JS, Powell BC, Cheney RE, A millenial myosin census, Mol Biol Cell 12 (2001) 780-94.

[Be] Berg HC, Constraints on models for the flagellar rotary motor, in [HuS], 491-501.

[Bo] Boyer PD, The ATP synthase - a splendid molecular machine, Annu Rev Biochem 66 (1997) 717-749.

[BKO] Bustamente C, Keller D, Oster G, The physics of molecular motors, Acc Chem Res 34 (2001) 412-420.

[CP] Cooke R, Pate E, The effects of ADP and phosphate on the contraction of muscle fibers, Biophys J 48 (1985) 789-798.

[Ei] Einstein A, Investigation on the theory of the Brownian movement (reprinted by Dover, 1985).

[El] Elston TC, Models of post-translational protein translocation, Biophys J 79 (2000) $2235-51$. 
[F+] Fisher AJ, Smith CA, Thoden JB, Smith R, Sutoh K, Holden HM, Rayment I, X-ray structures of the myosin motor domain of Dictyostelium discoideum complexed with $\mathrm{MgADP} \cdot \mathrm{BeF}_{x}$ and $\mathrm{MgADP} \cdot \mathrm{AlF}_{4}^{-}$, Biochem 34 (1995) 8960-72.

[FGR] Fortune NS, Geeves MA, Ranatunga KW, Pressure sensitivity of active tension in glycerinated rabbit psoas muscle fibres: effects of ADP and phosphate, J Muscle Res Cell Motil 10 (1989) 113-123.

[GKS] Givon D, Kupferman R, Stuart A, Extracting macroscopic dynamics: model problems and algorithms, Nonlinearity 17 (2004) R55-R127.

[GH] Geeves MA, Holmes KC, Structural mechanism of muscle contraction, Annu Rev Biochem 68 (1999) 687-728.

[HTB] Hänggi P, Talkner P, Borkovec M, Reaction-rate theory: fifty years after Kramers, Rev Mod Phys 62 (1990) 251-341.

[HMD] Herant M, Marganski WA, Dembo M, The mechanics of neutrophils: synthetic modelling of three experiments, Biophys J 84 (2003) 3389-3413.

[HMWZ] Hermans J, Mann G, Wang L, Zhang L, Simulation studies of protein-ligand interactions, in: Computational molecular dynamics: challenges, methods, ideas, eds Deuflhard P, Hermans J, Leimkuhler B, Mark AE, Reich S, Skeel RD (Springer, 1999), 129-148.

[Hill] Hill TL, Free energy transduction in biology (Academic, 1977).

[HE] Hill TL, Eisenberg E, Can free energy transduction be localized at some crucial part of the enzymatic cycle? Q Rev Biophys 14 (1981) 463-511.

[HK] Hill TL, Kirchner MW, Subunit treadmilling of microtubules or actin in the presence of cellular barriers: possible conversion of chemical free energy into mechanical work, Proc Nat Acad Sci USA 79 (1982) 490-4.

[H+] Holmes KC, Angert I, Kull FJ, Jahn W, Schröder RR, Electron cryo-microscopy shows how strong binding of myosin to actin releases nucleotide, Nature 425 (2003) 423-7.

[HTS] Holmes KC, Trentham DR, Simmons R (eds), Myosin, muscle and motility, Phil Trans Roy Soc B 359 (2004) 1811-1964.

[HoS] Houdusse A, Sweeney HL, Myosin motors: missing structures and hidden springs, Curr Opin Struct Biol 11 (2001) 182-194.

[How] Howard J, Mechanics of motor proteins and the cytoskeleton (Sinauer, 2001).

[HuS] Huxley AF, Simmons RM (eds), The molecular physics of biological movement, Phil Trans Roy Soc Lond B 355 (2000) 413-545.

[KS] Keener J, Sneyd J, Mathematical Physiology (Springer, 1998).

[Ko] Kodama T, Physiol Rev 65 (1985) 467-551.

[LL] Landau L, Lifshitz EM, Statistical Physics Part 1, (Pergamon Press, 1980).

[L] Lauffer MA, Entropy-driven processes in biology (Springer, 1975).

[MSXK] Ma J, Sigler PB, Xu Z, Karplus M, A dynamic model for the allosteric mechanism of GroEL, J Mol Bio 302 (2000) 303-313. 
[M] MacKay RS, Chaos in three physical systems, to appear in Equadiff 2003, eds F Dumortier, H Broer, J Mawhin, A Vanderbauwhede, S Verduyn Lunel (World Sci, 2005) 59-72; and Supplement to "Chaos in three physical systems, Section 3: Ergodic Pumping", http://www.maths.warwick.ac.uk/ mackay/publications.html

[MM] MacKay RS, MacKay DJC, Ergodic pumping: a proposed mechanism for the power stroke of myosin, abstract P3, Roy Soc Chem Chemical Biology Forum on "Biomolecular dynamics and force generation", 4-6 Sept 2003.

[MC+] Malnasi-Csizmadia A, Pearson DS, Kovacs M, Wooley RJ, Geeves MA, Bagshaw $\mathrm{CR}$, Kinetic resolution of a conformational transition and the ATP hydrolysis step using relaxation methods with a Dictyostelium myosin II mutant containing a single tryptophan residue, Biochem 40 (2001) 12727-37.

[MB] Marrink S-J, Berendsen HJC, Simulation of water transport through a lipid membrane, J Phys Chem 98 (1994) 4155-68.

[MK] McCammon JA, Karplus M, The dynamic picture of protein structure, Acc Chem Res 16 (1983) 187-193.

[MHG] Millar NC, Howarth JV, Gutfreund H, A transient kinetic study of enthalpy changes during the reaction of myosin subfragment 1 with ATP, Biochem J 248 (1987) 683-690.

[NMW] Nicholls JG, Martin AR, Wallace BG, From neurons to brain (Sinauer, 3rd ed, 1992).

[NFG] Nosek TM, Fender KY, Godt RE, Influence of inorganic phosphate $\left(P_{i}\right)$ and related compounds on contraction of skinned muscle fibres, M-AM-A9, Biophys J 51 (1987) 5 a.

[O] Oksendal B, Stochastic differential equations (Springer, 4th ed, 1995).

[PJ] Page ML, Jencks WP, Entropic contributions to rate accelerations in enzymatic and intramolecular interactions and the chelate effect, Proc Nat Acad Sci USA 68 (1971) 1678-83.

[QH] Qian H, Hopfield JJ, Entropy-enthalpy compensation: perturbation and relaxation in thermodynamic systems, J Chem Phys 105 (1996) 9292-8.

[R] Reimann P, Brownian motors: noisy transport far from equilibrium, Phys Rep 361 (2002) 57-265.

[Re+] Reubold TF, Eschenburg S, Becker A, Kull FJ, Manstein DJ, A structural model for actin-induced nucleotide release in myosin, Nature Struct Biol 10 (2003) 826-830.

[S+] Simpson AA, Tao Y, Leiman PG, Badasso MO, He Y, Jardine PJ, Olson NH, Morais MC, Grimes S, Anderson DL, Baker TS, Rossmann MG, Structure of the bacteriophage $\phi 29$ DNA packaging motor, Nature 408 (2000) 745-50.

[SG] Smith DA, Geeves MA, Strain-dependent cross-bridge cycle for muscle, parts I and II, Biophys J 69 (1995) 524-537, 538-552.

[SS] Smith DA, Sleep J, Mechanokinetics of rapid tension recovery in muscle: the myosin working stroke is followed by slower release of phosphate, Biophys J 87 (2004) 442-456.

[SH] Sweeney HL, Houdusse A, The motor mechanism of myosin V: insights for muscle contraction, in [HTS], 1829-42. 
[TSG] Takagi Y, Shuman H, Goldman YE, Coupling between phosphate release and force generation in muscle actomyosin, in [HTS], 1913-20.

[Va] Vale RD, Switches, latches and amplifiers: common themes of G proteins and molecular motors, J Cell Biol 135 (1996) 291-302.

[VM] Vale RD, Milligan RA, On-line supplement to "The way things move: looking under the hood of molecular motor proteins", Science 288 (2000) 88-95.

[W+] Wang HW, Chen Y, Yang H, Chen X, Duan M-X, Tai PC, Sui S-F, Ring-like pore structures of SecA, Proc Nat Acad Sci USA 100 (2003) 4221-6.

[WS] Wriggers W, Schulten K, Biophys J 73 (1997) 624 and (1998) (or do I mean Proteins 35 (1999) 262-73?)

[YLR] Yount RG, Lawson D, Rayment I, Is myosin a "back door" enzyme? Biophys J 68 (1995) 44s-49s

[Z+] Zeng W, Conibear PB, Dickens JL, Cowie RA, Wakelin S, Malnasi-Csizmadia, Bagshaw CR, Dynamics of actomyosin interaction in relation to the cross-bridge cycle, in [HTS], 1843-56.

Version 3.0 